\title{
Estimation and uncertainty quantification of optical properties directly from the photoacoustic time series
}

\author{
Aki Pulkkinen $^{\mathrm{a}}$, Ben T. Cox ${ }^{\mathrm{b}}$, Simon R. Arridge ${ }^{\mathrm{c}}$, Jari P. Kaipio ${ }^{\mathrm{a}, \mathrm{d}}$, and Tanja Tarvainen ${ }^{\mathrm{a}, \mathrm{c}}$ \\ ${ }^{a}$ University of Eastern Finland, Department of Applied Physics, P.O. Box 1627, 70211 Kuopio, \\ Finland \\ ${ }^{\mathrm{b}}$ University College London, Department of Medical Physics and Biomedical Engineering, \\ Gower Street, London WC1E 6BT, United Kingdom \\ ${ }^{\mathrm{c}}$ University College London, Department of Computer Science, Gower Street, London WC1E \\ 6BT, United Kingdom \\ ${ }^{\mathrm{d}}$ Department of Mathematics at University of Auckland, and Dodd-Walls Centre for Photonic \\ and Quantum Technologies, Private Bag 92019, Auckland Mail Centre, Auckland 1142, New \\ Zealand
}

\begin{abstract}
Quantitative photoacoustic tomography seeks to estimate the optical parameters of a target given photoacoustic measurements as a data. Conventionally the problem is split into two steps: 1) the acoustical inverse problem of estimating the acoustic initial pressure distribution from the acoustical time series data; 2) the optical inverse problem of estimating the optical absorption and scattering from the initial pressure distributions. In this work, an approach for estimating the optical absorption and scattering directly from the acoustical time series is investigated with simulations. The work combines a homogeneous acoustical forward model, based on the Green's function solution of the wave equation, and a finite element method based diffusion approximation model of light propagation into a single forward model. This model maps the optical parameters of interest into a time domain signal. The model is used with a Bayesian approach to ill-posed inverse problems to form estimates of the posterior distributions for the parameters of interest. In addition to being able to provide point estimates of the parameters of interest, i.e. reconstruct the absorption and scattering distributions, the approach can be used to derive information on the uncertainty associated with the estimates.
\end{abstract}

Keywords: Bayesian methods, biomedical optical imaging, inverse problems, photoacoustic effects, tomography, ultrasonic imaging

\section{INTRODUCTION}

Quantitative photoacoustic tomography (QPAT) seeks to estimate the optical properties of an imaged target based on acoustic measurements obtained from the boundary. ${ }^{1}$ This inverse problem is often solved in two phases. $^{2,3}$ In the first phase, one estimates the initial pressure distribution based on the photoacoustic time series (acoustic inverse problem), ${ }^{4-7}$ corresponding to traditional photoacoustic tomography (PAT) ${ }^{8-10}$ and in the second phase, forms estimates for the optical parameters based on the absorbed energy density distribution (optical inverse problem), ${ }^{11-20}$ which can be obtained from the initial pressure distribution under certain conditions (e.g. known Grüneisen parameter). Due to the ill-posedness of the optical inverse problem, estimation of the optical parameters can be difficult. The problem is especially problematic when the acoustic measurements have been obtained in a acoustically limited view setting, resulting in significant distortions in the estimated initial pressure distributions. This can result in a subsequent failure in solving the optical inverse problem.

In this work, the QPAT inverse problem is solved by directly estimating the optical parameters from the acoustical boundary data, thus avoiding estimation of intermediate parameters, such as the initial pressure

Further author information: (Send correspondence to A.P.)

A.P: E-mail: Aki.Pulkkinen@uef.fi, Telephone: +358 503444908

Photons Plus Ultrasound: Imaging and Sensing 2017, edited by Alexander A. Oraevsky, Lihong V. Wang, Proc. of SPIE Vol. 10064, 100643N · (c) 2017 SPIE · CCC code: 1605-7422/17/\$18 - doi: 10.1117/12.2248837 
distribution. The inverse problem is solved using a Bayesian approach. ${ }^{21,22}$ Previously the direct estimation approach has been applied in Refs 23-28. This work extends the approach to the case in which full spatial distributions of both the optical absorption and scattering are estimated. ${ }^{29}$ It is numerically demonstrated, that the estimation of the absorption and scattering is feasible even in the situation of acoustically limited view measurement geometry. In addition to the estimates, the approach is capable of providing uncertainties related to the the estimates.

\section{MATERIALS AND METHODS}

\subsection{Photoacoustic Model}

In this work, optical propagation of light is modelled using the diffusion approximation (DA) of the radiative transfer equation (RTE). The DA is defined in domain $\Omega \subset \mathbb{R}^{n}$ with a Robin type boundary condition as

$$
\left\{\begin{aligned}
-\nabla \cdot \kappa \nabla \Phi+\mu_{\mathrm{a}} \Phi=0, & r \in \Omega \\
\zeta_{n} \Phi+\frac{1}{2} \kappa \nabla \Phi \cdot \nu=s, & r \in \partial \Omega
\end{aligned}\right.
$$

where $\Phi=\Phi(r)$ is the fluence (or photon density), $\kappa=\kappa(r)=\left(n\left(\mu_{\mathrm{a}}+\mu_{\mathrm{s}}^{\prime}\right)\right)^{-1}$ is the optical diffusion, $\mu_{\mathrm{a}}=\mu_{\mathrm{a}}(r)$ and $\mu_{\mathrm{s}}^{\prime}=\mu_{\mathrm{s}}^{\prime}(r)$ are the optical absorption and scattering, $\zeta_{n}$ is a dimension dependent scaling parameter $\left(\zeta_{2}=\right.$ $\left.\pi^{-1}, \zeta_{3}=4^{-1}\right), \nu$ is the outward normal on boundary $\partial \Omega$, and $s=s(r)$ is the illumination pattern, or inward light current (i.e. light source), on the boundary. ${ }^{30,31}$ The absorption of fluence results in an absorbed energy density field defined as

$$
H(r)=\mu_{\mathrm{a}}(r) \Phi(r)
$$

The acoustical propagation is modelled using a homogeneous, non-attenuating, free space, linear wave equation, equipped with initial conditions as

$$
\left\{\begin{aligned}
\frac{1}{c^{2}} \frac{\partial^{2} p}{\partial t^{2}} & =\nabla^{2} p, \quad r \in \mathbb{R}^{n} \\
p(r, t=0) & =p_{0}(r) \\
\frac{\partial}{\partial t} p(r, t=0) & =0
\end{aligned}\right.
$$

where $c$ is the speed of sound and $p_{0}(r)$ is the initial pressure distribution, created as a result of photoacoustic effect. ${ }^{32}$ The initial pressure distribution is defined as

$$
p_{0}(r)= \begin{cases}\gamma(r) H(r), & r \in \Omega \\ 0, & \text { otherwise }\end{cases}
$$

where $\gamma(r)$ is the Grüneisen parameter, and $H(r)$ is given by (2). In this work, value of $\gamma(r)=1$ is used throughout.

Observations of the acoustic field, $p(r, t)$, as a result of illumination $s(r)$ for a target with optical parameters $\left(\mu_{\mathrm{a}}(r), \mu_{\mathrm{s}}^{\prime}(r)\right)$, are modelled as being measured at discrete positions $r_{k}$ for duration of $T$. The measurements are denoted as

$$
p_{k}(t)=p\left(r_{k}, t\right), \quad k=1, \ldots, K, \quad t \in[0, T] .
$$

The optical model is solved using the finite element method (FEM), and the acoustical model is solved using a Green's function based approach. For details on the implementation of the photoacoustic model, see Ref 29. After discretizing the models, the optical parameters are expressed as

$$
\mu_{\mathrm{a}}(r) \approx \sum_{q=1}^{Q} \mu_{\mathrm{a}, q} \phi_{q}(r), \quad \mu_{\mathrm{s}}^{\prime}(r) \approx \sum_{q=1}^{Q} \mu_{\mathrm{s}, q}^{\prime} \phi_{q}(r)
$$


where $\phi_{q}(r)$ are the $Q$ (spatial) basis functions of the FE discretization, and $\mu_{\mathrm{a}, q}$ and $\mu_{\mathrm{s}, q}^{\prime}$ are the discrete representations of $\mu_{\mathrm{a}}$ and $\mu_{\mathrm{s}}^{\prime}$ respectively. The discretized forward model, corresponding to (1)-(5), for illumination pattern $s(r)=s_{m}(r)$ can be denoted as

$$
z_{m}=f_{m}(x)
$$

where $z_{m}=\left(p_{1}\left(t_{1}\right), \ldots, p_{1}\left(t_{L}\right), \ldots, p_{K}\left(t_{1}\right), \ldots, p_{K}\left(t_{L}\right)\right)^{\top} \in \mathbb{R}^{K L}$ is the measured photoacoustic time series, at positions $r_{k}, k=1, \ldots, K$, at time instances $t_{l}, l=1, \ldots, L, x=\left(\mu_{\mathrm{a}, 1}, \ldots, \mu_{\mathrm{a}, Q}, \mu_{\mathrm{s}, 1}^{\prime}, \ldots, \mu_{\mathrm{s}, Q}^{\prime}\right)^{\top} \in \mathbb{R}^{2 Q}$ is a vector of optical parameters, and $f_{m}(x): \mathbb{R}^{2 Q} \rightarrow \mathbb{R}^{K L}$ maps the optical parameters and the illumination pattern into the photoacoustic time series. For multiple illumination patterns $s_{m}, m=1, \ldots, M$, the forward model becomes

$$
z=f(x)
$$

with $z=\left(z_{1}^{\top}, \ldots, z_{M}^{\top}\right)^{\top} \in \mathbb{R}^{M K L}$, and $f(x)=\left(f_{1}(x)^{\top}, \ldots, f_{M}(x)^{\top}\right)^{\top}: \mathbb{R}^{2 Q} \rightarrow \mathbb{R}^{M K L}$.

\subsection{Inverse Problem}

In this work, the photoacoustic inverse problem is solved using a Bayesian approach. ${ }^{21,22}$ The observation model, polluted with additive noise $e$, is defined as

$$
y=f(x)+e,
$$

where $y$ represents noisy observations of $z$, and $f(x)$ is the deterministic forward model (8). In this work, the additive noise is assumed to be normally distributed as $e \sim \mathcal{N}\left(\eta_{e}, \Gamma_{e}\right)$. The prior information of the unknown optical parameters is similarly assumed to be normally distributed as $x \sim \mathcal{N}\left(\eta_{x}, \Gamma_{x}\right)$, and $x$ and $e$ are assumed to be mutually independent. Thus, the posterior distribution of the observation model (9) can be expressed as

$$
\pi(x \mid y) \propto \pi_{x}(x) \pi_{e}(y-f(x)),
$$

where $\pi(x \mid y), \pi_{x}(x)$, and $\pi(y \mid x)=\pi_{e}(y-f(x))$ are the probability density functions of the posterior, prior, and the likelihood distributions respectively. The maximum a posteriori (MAP) estimate of (10) can be obtained by solving a minimization problem

$$
x_{\mathrm{MAP}}=\underset{x}{\arg \min }\left\|L_{e}\left(y-f(x)-\eta_{e}\right)\right\|^{2}+\left\|L_{x}\left(x-\eta_{x}\right)\right\|^{2},
$$

where $L_{e}$ and $L_{x}$ are Cholesky decompositions, such that $\Gamma_{e}^{-1}=L_{e}^{\top} L_{e}$ and $\Gamma_{x}^{-1}=L_{x}^{\top} L_{x}$.

\subsection{Approximating the Uncertainty of the Estimate}

The uncertainty of the estimate is approximated by obtaining the MAP estimate, $x_{\mathrm{MAP}}$, with (11), and then approximating the observation model (9) with a linearized observation model (truncated Taylor series developed around $x_{\mathrm{MAP}}$ )

$$
y=f_{0}+J_{f}\left(x-x_{\mathrm{MAP}}\right)+e,
$$

where $f_{0}=f\left(x_{\mathrm{MAP}}\right)$, and $J_{f}=J_{f}\left(x_{\mathrm{MAP}}\right)$ is the Jacobian matrix of the forward model $f(x)$ evaluated at $x_{\mathrm{MAP}}$. The approximate posterior distribution for $x$, based on linearized observation model (12), can then be expressed as

$$
x \mid y \sim \mathcal{N}(\eta, \Gamma), \quad \eta=x_{\mathrm{MAP}}, \quad \Gamma=\left(\Gamma_{x}^{-1}+J_{f}^{\top} \Gamma_{e}^{-1} J_{f}\right)^{-1},
$$

where $\eta$ is the point estimate of the unknown optical parameters, and $\Gamma$ is its covariance matrix describing the uncertainty related to the estimates. From the covariance matrix, nodal standard deviations for the estimate can be computed by taking the square root of the elements on the diagonal, that is, for vector $\eta=\left(\eta_{1}, \ldots, \eta_{2 Q}\right)^{\top}$, standard deviation corresponding to $\eta_{q}$ is $\sigma_{q}=\sqrt{\Gamma_{q q}}$. 


\section{NUMERICAL SIMULATIONS}

\subsection{Simulation of the Data}

The simulation domain $\Omega$ was a two dimensional origin centered circle with diameter of 5 mm, discretized into 1176 triangular elements and 631 grid nodes. Two illumination patterns $(M=2)$ were used to generate the simulated data and were defined as

$$
s_{1}(r)=\frac{1}{2}(1+\cos (2 \theta)), \quad s_{2}(r)=\frac{1}{2}(1-\cos (2 \theta)),
$$

where $\theta$ is the polar angle of the boundary. Acoustical sensors were located on an origin centered circle of diameter $6 \mathrm{~mm}$. Five sensor configurations were simulated: I) $K=180$ sensors spanning polar angles [0,2 $\pi$, II) $K=90$ sensors spanning $\left[0,2 \pi[\right.$, III) $K=90$ sensors spanning [0, $\pi]$, IV) $K=90$ sensors spanning $\left[0, \frac{1}{2} \pi\right] \cup\left[\pi, \frac{3}{2} \pi\right]$, and V) $K=45$ sensors spanning $\left[0, \frac{1}{2} \pi\right]$. Speed of sound $c=1500 \mathrm{~m} / \mathrm{s}$ was used, and the data was simulated for duration of $T=6 \mu \mathrm{s}$ (sampling rate of $25.5 \mathrm{MHz}, 154$ samples) corresponding to acoustic propagation distance of $9 \mathrm{~mm}$. The optical absorption $\mu_{\mathrm{a}}$ and scattering $\mu_{\mathrm{s}}^{\prime}$, that were used to simulate the data, are shown in Figure 1. The figure also shows the corresponding fluence, initial pressure distribution, and the photoacoustic time series.

After simulating the photoacoustic time series, normally distributed noise with zero mean and standard deviation of $1 \%$ of the peak-to-peak observed pressure amplitude was added to obtain the noisy synthetic data used in the inversion.

\subsection{Parameters for the Inverse Problem}

In the inversion approach, a triangular grid composed of 1337 elements and 714 grid nodes was used to avoid an inverse crime.

The true values for the statistical parameters of the noise were used for the likelihood in the inversion. This corresponds to an assumption of a well characterized noise behavior of the imaging system.

The prior, used in this work is expressed as

$$
x \sim \mathcal{N}\left(\eta_{x}, \Gamma_{x}\right), \quad \eta_{x}=\left(\begin{array}{c}
\eta_{\mu_{\mathrm{a}}} \\
\eta_{\mu_{\mathrm{s}}^{\prime}}
\end{array}\right), \quad \Gamma_{x}=\left(\begin{array}{cc}
\Gamma_{\mu_{\mathrm{a}}} & 0 \\
0 & \Gamma_{\mu_{\mathrm{s}}^{\prime}}
\end{array}\right)
$$



$\mu_{\mathrm{s}}^{\prime}$



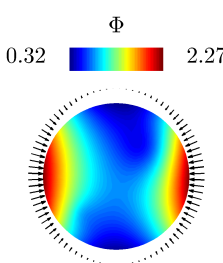





$p_{0}$

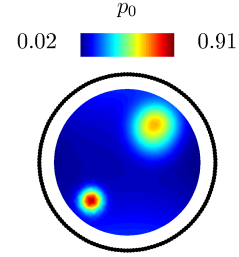



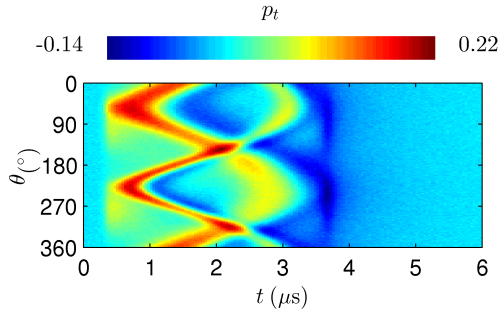

Figure 1. The optical absorption $\mu_{\mathrm{a}}$ (top of the first column) and scattering $\mu_{\mathrm{s}}^{\prime}$ (bottom of the first column) used to simulate the data, optical fluence $\Phi$ for the two illuminations used ( $s_{1}$ in the top and $s_{2}$ in the bottom of the second column: the black vectors denote the illumination amplitude on the boundary), initial pressure distributions $p_{0}$ (top and bottom of the third column: the black points denote locations for acoustical sensors in configuration I, $K=180$ sensors spanning $\left[0,2 \pi\left[\right.\right.$ ), photoacoustic time series $p_{t}$ as a function of time $t$ and acoustic sensors position in polar angle $\theta$ (top and bottom of the fourth column). 
where it has been assumed that $\mu_{\mathrm{a}} \sim \mathcal{N}\left(\eta_{\mu_{\mathrm{a}}}, \Gamma_{\mu_{\mathrm{a}}}\right), \mu_{\mathrm{s}}^{\prime} \sim \mathcal{N}\left(\eta_{\mu_{\mathrm{s}}^{\prime}}, \Gamma_{\mu_{\mathrm{s}}^{\prime}}\right)$, and $\mu_{\mathrm{a}} \perp \mu_{\mathrm{s}}^{\prime}$. For parameters of the priors for optical absorption and scattering, the following values are used:

$$
\eta_{\mu_{\mathrm{a}}}=\frac{1}{2}\left(\max \mu_{\mathrm{a}, \mathrm{TRUE}}+\min \mu_{\mathrm{a}, \mathrm{TRUE}}\right), \quad \eta_{\mu_{\mathrm{s}}^{\prime}}=\frac{1}{2}\left(\max \mu_{\mathrm{s}, \mathrm{TRUE}}^{\prime}+\min \mu_{\mathrm{s}, \mathrm{TRUE}}^{\prime}\right),
$$

and

$$
\Gamma_{\mu_{\mathrm{a}}}=\frac{1}{4}\left(\max \mu_{\mathrm{a}, \mathrm{TRUE}}-\min \mu_{\mathrm{a}, \mathrm{TRUE}}\right)^{2} \Xi, \quad \Gamma_{\mu_{\mathrm{s}}^{\prime}}=\frac{1}{4}\left(\max \mu_{\mathrm{s}, \mathrm{TRUE}}^{\prime}-\min \mu_{\mathrm{s}, \mathrm{TRUE}}^{\prime}\right)^{2} \Xi,
$$

where $\Xi$ is a matrix defined by exponential covariance function

$$
\Xi_{i j}=\exp \left(-\left\|r_{i}-r_{j}\right\| / \xi\right)
$$

where $r_{i}, r_{j} \in \Omega$, and $\xi>0$ is the characteristic length describing a distance over which the estimated parameter is expected to have significant correlation. The choices (15)-(18) are equivalent to (prior) expectation that the values of $\mu_{\mathrm{a}}$ and $\mu_{\mathrm{s}}^{\prime}$ are with probability of $68.2 \%$ within intervals [max $\mu_{\mathrm{a}, \mathrm{TRUE}}, \min \mu_{\mathrm{a}, \mathrm{TRUE}}$ ] and

$\left[\max \mu_{\mathrm{s}, \mathrm{TRUE}}^{\prime}, \min \mu_{\mathrm{s}, \mathrm{TRUE}}^{\prime}\right]$, and that spatially the optical parameters are expected to have shapes that can be described by length scale $\xi$. In the inversion, the true minimum and maximum of the absorption and scattering were used to define the prior parameters. This corresponds to a choice of a good quantitative prior. For more discussion on the choice of the prior in QPAT, see. ${ }^{19}$

The minimization (optimization) problem (11), to obtain the MAP estimates, was solved using a GaussNewton algorithm augmented with a line search algorithm. ${ }^{33}$

\subsection{Numerical Results}

Figure 2 shows the MAP estimates of the optical absorption and scattering for various acoustic measurement settings and the standard deviations of the estimates. Visually (qualitatively) the MAP estimates of absorption look similar to true distributions in situations where the acoustical sensors have been spread to cover the entire circle. When the sensors cover less than half of the circle, streaks appear in the estimates of the absorption. Standard deviations (uncertainties) of the absorption increase in amplitude as the number of sensors is reduced, and the spatial distribution of the deviations increases further away from the enclosure of the sensors.

The MAP estimates of the scattering are visually very similar to each other for the acoustical measurement settings where the sensors cover at least half of the circle. A similar remark can be made for the standard deviation of the scattering. For the situation where only quarter of the circle is covered, the MAP estimate deviates more from the true parameter. Unlike in the uncertainty of the absorption, the uncertainties of the scattering do not seem to depend as strongly on the placement or the number of acoustical sensors.

\section{CONCLUSIONS}

Quantitative estimates and uncertainties of optical absorption and scattering from photoacoustic time series were estimated using a Bayesian approach. Using this approach, it was possible to obtain quantitatively accurate estimates of both absorption and scattering in acoustically limited view setting. Based on these results, it is apparent that the width (uncertainty) of the posterior distribution of absorption increases when the number of acoustical sensors is reduced. The shape of the posterior distribution is likewise affected by the acoustic sensor configuration. The impact of the acoustic sensor locations on estimates and uncertainty of scattering is not as significant as they are on absorption.

\section{ACKNOWLEDGMENTS}

This work has been supported by the Academy of Finland (projects 286247, 250215 Finnish Centre of Excellence in Inverse Problems Research). 


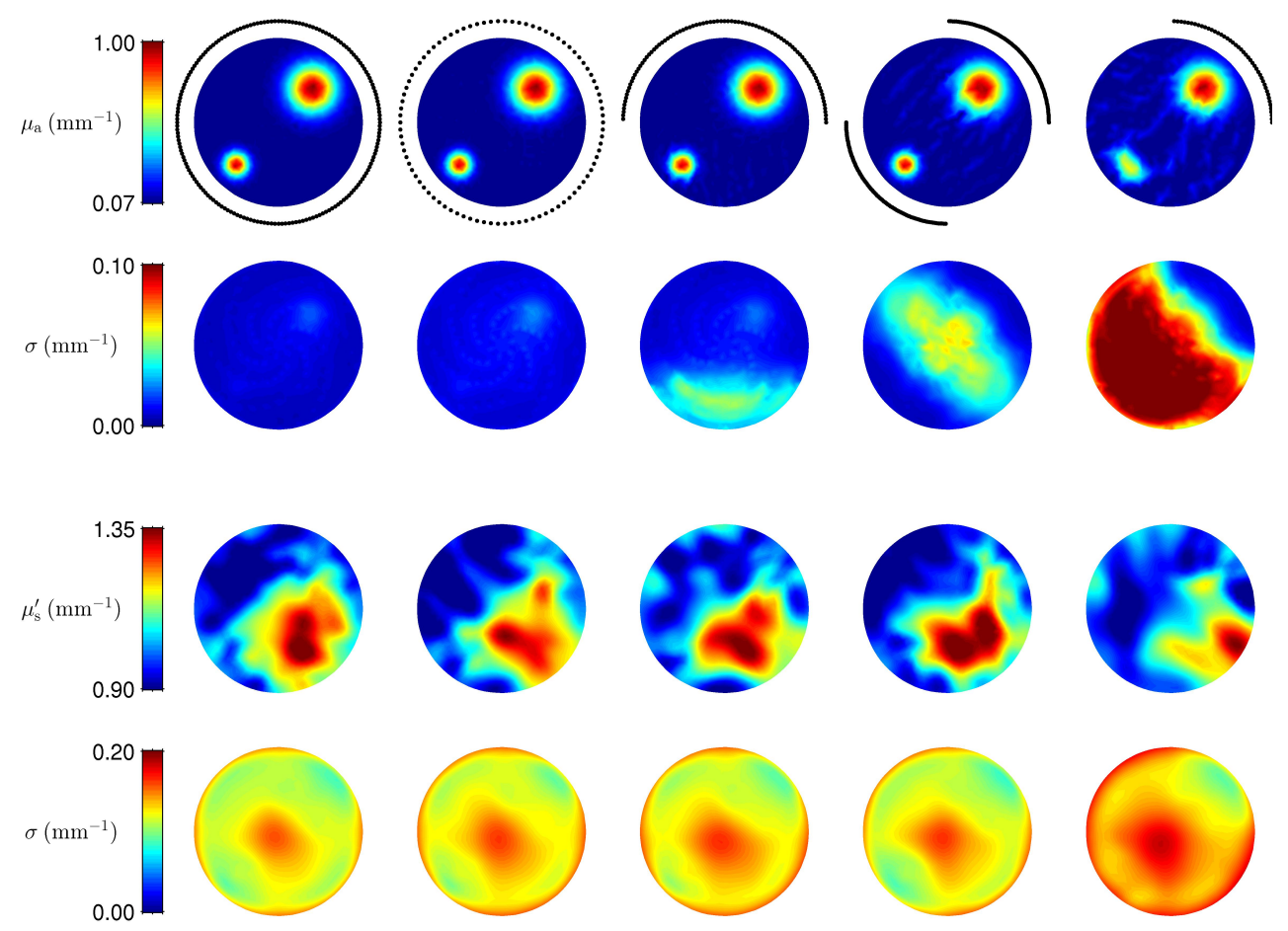

Figure 2. The MAP estimates $\mu_{\mathrm{a}}$ and $\mu_{\mathrm{s}}^{\prime}$ (first and third row respectively) for the acoustic sensor configurations I-V (from left to right). The black points denote the acoustic sensors. Below the estimates, (approximate) standard deviation of the estimates (second and fourth row corresponding to $\mu_{\mathrm{a}}$ and $\mu_{\mathrm{s}}^{\prime}$ respectively).

\section{REFERENCES}

[1] Cox, B., Laufer, J. G., Arridge, S. R., and Beard, P. C., "Quantitative spectroscopic photoacoustic imaging: a review," J. Biomed. Opt. 17(6), 061202 (2012).

[2] Cox, B. T., Laufer, J. G., and Beard, P. C., "The challenges for quantitative photoacoustic imaging," Proc. SPIE 7177, 717713 (2009).

[3] Tarvainen, T., Pulkkinen, A., Cox, B. T., Kaipio, J. P., and Arridge, S. R., "Bayesian image reconstruction in quantitative photoacoustic tomography," IEEE Trans. Med. Imag. 32(12), 2287-98 (2013).

[4] Xu, Y. and Wang, L. V., "Reconstructions in limited-view thermoacoustic tomography," Med. Phys. 31(4), $724-33$ (2004).

[5] Xu, M. and Wang, L. V., "Universal back-projection algorithm for photoacoustic computed tomography," Phys. Rev. E 71(1), 016706 (2005).

[6] Kuchment, P. and Kunyansky, L., "Mathematics of thermoacoustic tomography," Eur. J. Appl. Math. 19(2), 191-224 (2008).

[7] Tick, J., Pulkkinen, A., and Tarvainen, T., "Image reconstruction with uncertainty quantification in photoacoustic tomography," J. Acoust. Soc. Am. 139(4), 1951-1961 (2016).

[8] Wang, L. V., "Prospects of photoacoustic tomography," Med. Phys. 35(12), 5758-67 (2008).

[9] Li, C. and Wang, L. V., "Photoacoustic tomography and sensing in biomedicine," Phys. Med. Biol. 54(19), R59-97 (2009).

[10] Beard, P., "Biomedical photoacoustic imaging," Interface Focus 5(1), 602-31 (2011). 
[11] Cox, B. T., Arridge, S. R., Köstli, K. P., and Beard, P. C., "Two-dimensional quantitative photoacoustic image reconstruction of absorption distributions in scattering media by use of a simple iterative method," Appl. Opt. 45(8), 1866-75 (2006).

[12] Zemp, R. J., "Quantitative photoacoustic tomography with multiple optical sources," Appl. Opt. 49(18), 3566-72 (2010).

[13] Bal, G. and Uhlmann, G., "Inverse diffusion theory of photoacoustics," Inv. Probl. 26(8), 085010 (2010).

[14] Bal, G., Jollivet, A., and Jugnon, V., "Inverse transport theory of photoacoustics," Inv. Probl. 26(2), 025011 (2010).

[15] Bal, G. and K., "Multi-source quantitative photoacoustic tomography in a diffusive regime," Inv. Probl. 27(7), 075003 (2011).

[16] Bal, G. and Ren, K., "On multi-spectral quantitative photoacoustic tomography in diffusive regime," Inv. Probl. 28(2), 025010 (2012).

[17] Tarvainen, T., Cox, B. T., Kaipio, J. P., and Arridge, S. R., "Reconstructing absorption and scattering distributions in quantitative photoacoustic tomography," Inv. Probl. 28(8), 084009 (2012).

[18] Saratoon, T., Tarvainen, T., Cox, B. T., and Arridge, S. R., "A gradient-based method for quantitative photoacoustic tomography using the radiative transfer equation," Inv. Probl. 29(7), 075006 (2013).

[19] Pulkkinen, A., Cox, B. T., Arridge, S. R., Kaipio, J. P., and Tarvainen, T., "A Bayesian approach to spectral quantitative photoacoustic tomography," Inv. Probl. 30(6), 065012 (2014).

[20] Pulkkinen, A., Cox, B. T., Arridge, S. R., Kaipio, J. P., and Tarvainen, T., "Quantitative photoacoustic tomography using illuminations from a single direction," J. Biomed. Opt. 20(3), 036015 (2015).

[21] Kaipio, J. P. and Somersalo, E., [Statistical and Computational Inverse Problems], Springer, New York (2005).

[22] Tarantola, A., [Inverse Problem Theory and Methods for Model Parameter Estimation], SIAM, Philadelphia (2005).

[23] Yuan, Z. and Jiang, H., "A calibration-free, one-step method for quantitative photoacoustic tomography," Med. Phys. 39(11), 6895-9 (2012).

[24] Song, N., Deumié, C., and Silva, A. D., "Considering sources and detectors distributions for quantitative photoacoustic tomography," Biomed. Opt. Express 5(11), 3960-74 (2014).

[25] Ding, T., Ren, K., and Vallélian, S., "A one-step reconstruction algorithm for quantitative photoacoustic imaging," Inv. Probl. 31(9), 095005 (2015).

[26] Haltmeier, M., Neumann, L., and Rabanser, S., "Single-stage reconstruction algorithm for quantitative photoacoustic tomography," Inv. Probl. 31(6), 065005 (2015).

[27] Gao, H., Feng, J., and Song, L., "Limited-view multi-source quantitative photoacoustic tomography," Inv. Probl. 31(6), 065004 (2015).

[28] Bal, G. and Moradifam, A., "Photo-acoustic tomography in a rotating measurement setting," Inv. Probl. 32(10), 105012 (2016).

[29] Pulkkinen, A., Cox, B. T., Arridge, S. R., Goh, H., Kaipio, J. P., and Tarvainen, T., "Direct estimation of optical parameters from photoacoustic time series in quantitative photoacoustic tomography," IEEE Trans. Med. Imag. 35(11), 2497-2508 (2016).

[30] Case, K. M. and Zweifel, P. F., [Linear Transport Theory], Addison-Wesley, Reading (1967).

[31] Ishimaru, A., [Wave Propagation and Scattering in Random Media vol. 1], Academic, New York (1978).

[32] Gusev, V. Z. and Karabutov, A. A., [Laser Optoacoustics], AIP, College Park (1992).

[33] Nocedal, J. and Wright, S. J., [Numerical Optimization], Springer, New York (1999). 